\title{
Surface-plasmon enhanced bright emission from CdSe quantum-dot nanocrystals
}

\author{
Koichi Okamoto, Saurabh Vyawahare, and Axel Scherer \\ Departments of Applied Physics and Physics, California Institute of Technology, Pasadena, California 91125
}

Received March 30, 2006; accepted April 4, 2006; posted April 21, 2006 (Doc. ID 69436)

We obtained very bright light emission from CdSe quantum dots (QDs) by using the surface-plasmon (SP) coupling technique. 23-fold enhanced photoluminescence (PL) intensities and two-fold increased PL decay rates are observed when the QDs are located on evaporated gold films. This enhancement is not effective for CdSe cores with $\mathrm{ZnS}$ shells (ZnS/CdSe). The reason for this difference can be explained by using the SP dispersion diagram and by considering the SP coupling mechanism. We discuss the inherent merits and demerits of this technique to increase the emission efficiency. This technique will enable high-speed and efficient light emission for optically as well as electrically pumped light emitters. (C) 2006 Optical Society of America OCIS codes: $240.6680,300.6500$.

\section{INTRODUCTION}

Solid-state light-emitting devices are expected to eventually replace more-traditional fluorescent tubes as illumination sources. One possibility is InGaN-based quantum wells (QWs), which are very bright light sources in UV, blue, green, and red emissions. ${ }^{1} \mathrm{CdSe}$-based quantum dot (QD) nanocrystals are also very promising materials for light-emitting sources. CdSe-based QD nanocrystals possess a number of advantageous features and have been used in light-emitting diodes (LEDs) $)^{2,3}$ and as biological fluorescent labels. ${ }^{4,5}$ However, their light-emission efficiencies are still substantially lower than those of fluorescent tubes. The most important requirement for a competitive solid-state light-emitting device is high quantum efficiency of light emission. Recently, ${ }^{6}$ we reported a method for enhancing the light-emission efficiency from InGaN QWs by controlling the energy transfer between QWs and surface plasmons (SPs). The idea of SPenhanced light emission was previously described ${ }^{7-12}$ and, for the first time (to our knowledge), we directly measured significant enhancement of internal quantum efficiency of emission due to spontaneous recombination rate increases. ${ }^{6,13}$ We believe that this SP-enhanced technique can be applied not only to InGaN-based materials but also to various other materials. Indeed, we have also reported similar light enhancement for dye-molecule doped polymer materials. ${ }^{14}$

In this paper, we investigate the direct observation of SP-coupled spontaneous emission from CdSe and CdSe/ZnS QDs by photoluminescence (PL) measurements. The emission rates are also measured by using the time-resolved PL measurements with a streak camera system. On the basis of these results, possible mechanisms and dynamics of the SP coupling and lightemission enhancement are considered.

\section{EXPERIMENT}

CdSe-based QDs were purchased from Evident Technologies. These QDs have an emission peak around $620 \mathrm{~nm}$ and a crystal diameter of approximately $5 \mathrm{~nm}$. The toluene solutions of the QDs were dispersed on quartz substrates. After the solutions evaporated, a monolayer of the QD nanocrystals remained on the substrates. The half parts of the quartz surface were covered by a $50 \mathrm{~nm}$ gold layer by thermal evaporation. The sample structure is shown in Fig. 1.

An InGaN diode laser (405 nm) was used to excite the samples. The PL signal was collected and focused into an optical fiber and subsequently detected with a spectrometer (10 $\mu \mathrm{m}$ slit width, 300 periods/mm grating) exit with a liquid-nitrogen-cooled CCD camera (Princeton Instruments).

To perform time-resolved PL measurements, a frequency-doubled beam of a mode-locked Ti:sapphire laser (Spectra Physics) was used to excite the samples. The pulse width, wavelength, and repetition rate were chosen as $100 \mathrm{fs}, 400 \mathrm{~nm}$, and $80 \mathrm{MHz}$, respectively. A Hamamatsu Photonics C4334 Streak Camera system with a time resolution of $5 \mathrm{ps}$ served as the detector.

\section{RESULTS AND DISCUSSION}

\section{A. Enhancement of PL Intensity}

Figure 2(a) shows the PL spectrum taken for the CdSe QDs with and without gold coating. A dramatic enhancement in the PL intensity from the QDs on gold layer was very clearly observed. When the PL peak of the QDs on quartz was normalized to 1 , a 23-fold increase of PL intensity was observed. This remarkable PL enhancement should be attributed to strong interaction with SP. The electron-hole pairs generated in the QDs couple to electron vibrations at the gold surface when the energies of electron-hole pairs in the QDs and of the metal SPs are similar. The propagation wave number $k$ of SP mode generated at the metal/dielectric interface is given by 


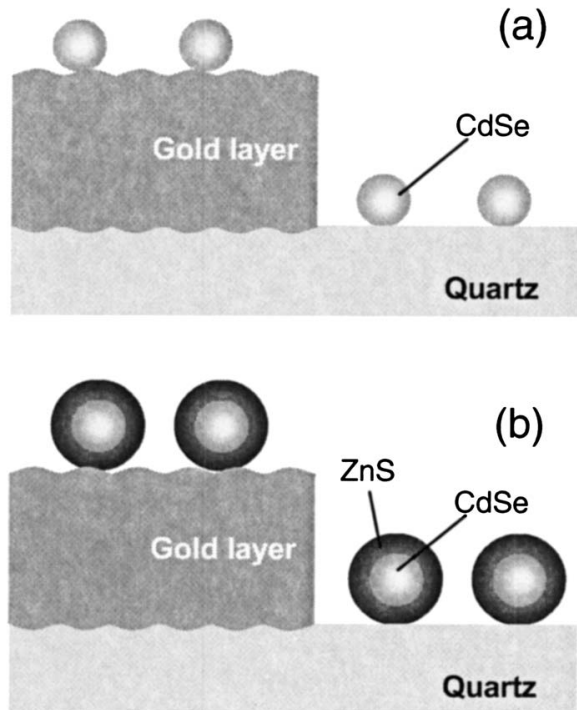

Fig. 1. Sample structures of (a) CdSe and (b) CdSe/ZnS nanocrystals on gold-coated quartz chips.
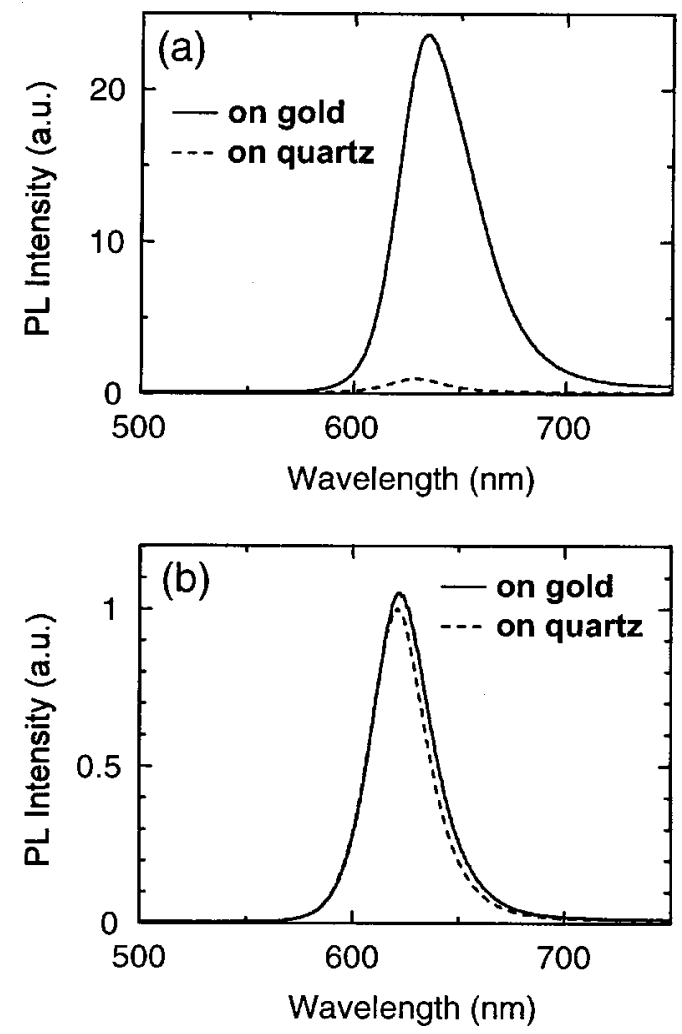

Fig. 2. PL spectra for (a) CdSe and (b) CdSe/ZnS nanocrystals on gold and quartz.

$$
k=\frac{\omega}{c}\left(\frac{\epsilon_{1} \epsilon_{2}}{\epsilon_{1}+\epsilon_{2}}\right)^{1 / 2}
$$

where $\omega$ is the frequency, $c$ is the speed of light, and $\epsilon_{1}$ and $\epsilon_{2}$ are the real dielectric constants of metal and dielectric material, respectively. The dielectric function of CdSe was already known. ${ }^{15}$ By the dispersion diagram calculated by Eq. (1), the SP frequency at the CdSe/gold interface is obtained as $\sim 2.2 \mathrm{eV}(\sim 570 \mathrm{~nm})$. This value is close to the obtained emission spectrum.

We investigated similar measurements for CdSe cores with $\mathrm{ZnS}$ shells as shown in Fig. 2(b). In this figure, the enhancement of PL intensity was not remarkable compared with the result of naked CdSe without shells. This discrepancy is an unexpected result, and elucidating the reason of this discrepancy should be useful for understanding the SP coupling mechanism and optimizing more-efficient coupling conditions. The SP frequency at the $\mathrm{ZnS} /$ gold interface is calculated as $\sim 2.3 \mathrm{eV}$ $(\sim 550 \mathrm{~nm})$ by the dielectric functions of CdSe. ${ }^{16}$ This value is slightly higher than the obtained PL peak compared with the result of naked CdSe, but the difference is only $\sim 20 \mathrm{~nm}$. The SP penetration depth $Z$ of the dielectric materials is given by

$$
Z=\frac{c}{\varpi}\left(\frac{\epsilon_{2}-\epsilon_{1}}{\epsilon_{1}{ }^{2}}\right)^{1 / 2} .
$$

The obtained penetration depth of $\mathrm{ZnSe}$ and $\mathrm{CdS}$ are $Z$ $=27$ and $33 \mathrm{~nm}$, respectively. Compared with these values, the thickness of $\mathrm{ZnS}$ shell layer is much thinner (a few nanometers). Such a very thin $\mathrm{ZnS}$ layer may not influence the SP frequency. This suggests that the difference of the SP frequency may not be a reason for the reduction of the SP enhancement for $\mathrm{ZnS} / \mathrm{ZnSe}$ sample.

The SP-enhanced luminescence of CdSe QDs has been reported by a few groups. Kulakovich et al. ${ }^{17}$ reported five-fold enhancement of the PL intensity for CdSe/ZnS QD and gold colloids. Song et al. ${ }^{18}$ achieved $\sim 50$-fold enhancement by using CdSe/ZnS QDs and nanoperiodic silver arrays fabricated by electron-beam lithography. Grycczynski et al. ${ }^{19}$ reported a well-polarized, directional, and photostable SP coupling emission by using CdSe/ZnS QDs on $\mathrm{SiO}_{2} /$ silver thin layers. Compared with these reports, our method is much simpler and easier. We used naked CdSe and an evaporated gold layer, but we obtained still remarkable enhancement (23-fold). Special geometry or nanoperiodic structures are not necessary for our method.

The importance of the metal-surface topography for the light extraction from the SP mode has been discussed. ${ }^{6}$ If the metal surface is perfectly flat, the SP energy will be thermally dissipated. However, the SP energy can be extracted as light by providing roughness or by nanostructuring the metal layer. Such roughness allows SPs of high momentum to scatter, lose momentum, and then couple to radiated light. The few tens of nanometer sized roughness in the gold surface layer can be obtained by controlling the evaporation conditions. We already described this fact in previous reports, ${ }^{6}$ and the roughness of the gold surface was actually observed with atomic force microscopy and the scanning electron microscopy. Thus we can obtain the remarkable SP-enhanced emission by using evaporated metal layers, without metal colloids or nanostructures.

\section{B. Enhancement of Spontaneous Emission Rate}

Enhancement of a spontaneous emission rate is also very important for the development of communication technology and optical computing. The SP-coupling technique has the potential to enhance the spontaneous-emission 

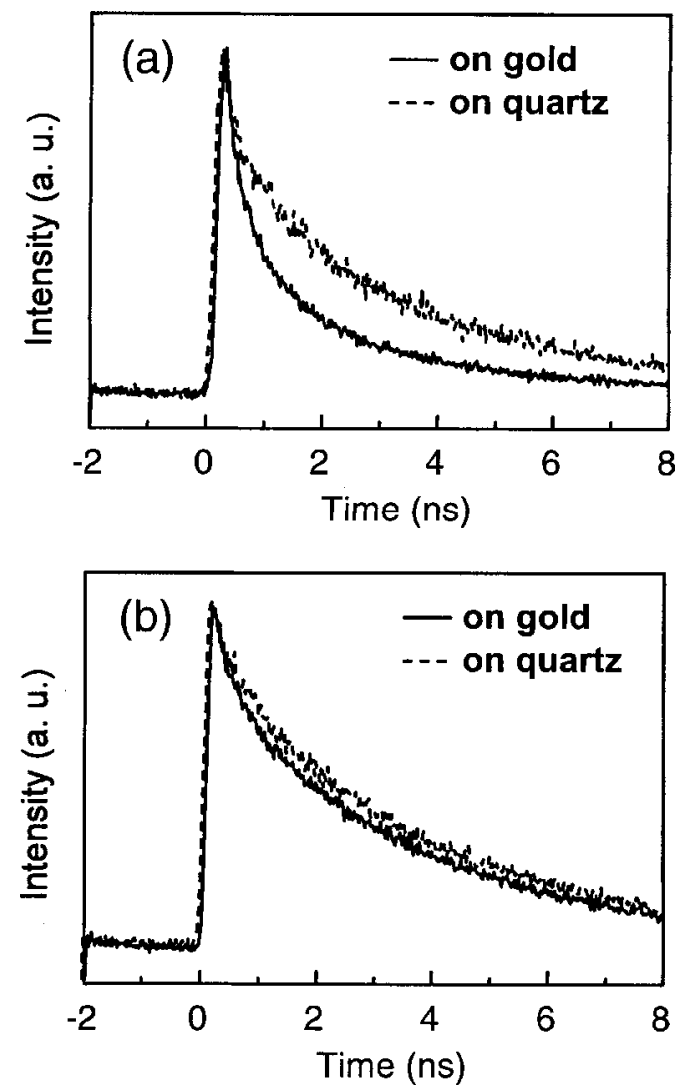

Fig. 3. Time profiles of integrated PL signal for (a) CdSe and (b) $\mathrm{CdSe} / \mathrm{ZnS}$ nanocrystals on gold and quartz.

rate. ${ }^{10-12}$ Very recently, we succeeded in direct observation of a dramatically enhanced spontaneous-emission rate of blue emission from InGaN/GaN with a silver layer. ${ }^{13}$ Figure 3 shows the PL time profiles of the QDs on gold and quartz taken for (a) naked CdSe and (b) $\mathrm{CdSe} / \mathrm{ZnS}$. For the naked CdSe case, clearly the PL decay of QDs on gold is faster than that of QDs on quartz. This suggests that the PL decay rates are also enhanced by the SP coupling. The time-resolved PL dynamics of CdSe QW had been discussed on the basis of carrier dynamics, for example, using the self-organized CdSe/ZnSe QDs on GaAs. ${ }^{20}$ Usually, electron-hole pairs generated in semiconductor materials decay through both radiative and nonradiative recombination processes. The PL decay rate $\left(k_{\mathrm{PL}}\right)$ is given by the radiative $\left(k_{\mathrm{rad}}\right)$ and nonradiative $\left(k_{\text {non }}\right)$ recombination rates as $k_{\mathrm{PL}}=k_{\text {rad }}+k_{\text {non }}$. The mechanism of the SP-enhanced PL decay rates were already reported for InGaN/GaN QW (Ref. 13). If the QD-SP coupling rate $\left(k_{\mathrm{SP}}\right)$ is much faster than $k_{\text {rad }}$ and $k_{\text {non }}$, the $\mathrm{PL}$ decay rate with SP coupling $\left(k_{\mathrm{PL}}{ }^{*}=k_{\mathrm{rad}}+k_{\mathrm{non}}+k_{\mathrm{SP}}\right)$ should be much faster than $k_{\mathrm{PL}}$. High electromagnetic fields are introduced by the large density of states from the SP dispersion diagram, and these fields increase the QD-SP coupling rate. On the other hand, the PL profiles of CdSe/ZnS are not so different on gold and on quartz. This tendency corresponds to the results of the intensities of the PL spectra.

The obtained PL time profiles were fitted to single exponential functions and the PL decay rates were obtained at every wavelength. The obtained $k_{\mathrm{PL}}$ and $k_{\mathrm{PL}}{ }^{*}$ values were plotted against wavelength in Fig. 4 for (a) naked $\mathrm{CdSe}$ and (b) CdSe/ZnS. $k_{\mathrm{PL}}$ values of CdSe on quartz were almost constant $\left(\sim 0.5 \mathrm{~ns}^{-1}\right)$ at every wavelength. $k_{\mathrm{PL}}{ }^{*}$ values of CdSe on gold were larger than $k_{\mathrm{PL}}{ }^{*}$ and become even larger at the shorter wavelength. This tendency is reasonable, because the SP frequency at CdSe/ quartz $(\sim 570 \mathrm{~nm})$ is slightly shorter than the PL spectrum. Thus SP coupling should be more effective at the shorter wavelength. On the other hand, both $k_{\mathrm{PL}}$ and $k_{\mathrm{PL}}{ }^{*}$ values of $\mathrm{CdSe} / \mathrm{ZnS}$ were similar at every wavelength.

Another remarkable tendency is that both $k_{\mathrm{PL}}$ and $k_{\mathrm{PL}}{ }^{*}$ values of $\mathrm{CdSe} / \mathrm{ZnS}$ were much smaller than those of $\mathrm{CdSe}$ at the longer wavelength region and become larger at the shorter wavelength. This behavior can be explained by using the carrier localization model. The bandgap of $\mathrm{ZnS}(3.68 \mathrm{eV})$ is much higher than that of CdSe $(1.74 \mathrm{eV})$. The generated carriers are strongly localized in the CdSe core in the $\mathrm{ZnS}$ shell. This prevents the carriers from being captured in the nonradiative recombination center (defects, dislocation, impurity, etc.). Thus the $k_{\mathrm{PL}}$ values at the longer wavelength are very small. On the other hand, the $k_{\text {PL }}$ values at the shorter wavelength are very large, because they include the carrier localization process into lower energy level.

\section{Purcell Enhancement Factor}

The Purcell enhancement factor $\left(F_{p}\right)^{21}$ quantifies the increase in spontaneous-emission rate into a mode of interest and is obtained by the ratios of the original and enhanced PL lifetimes as
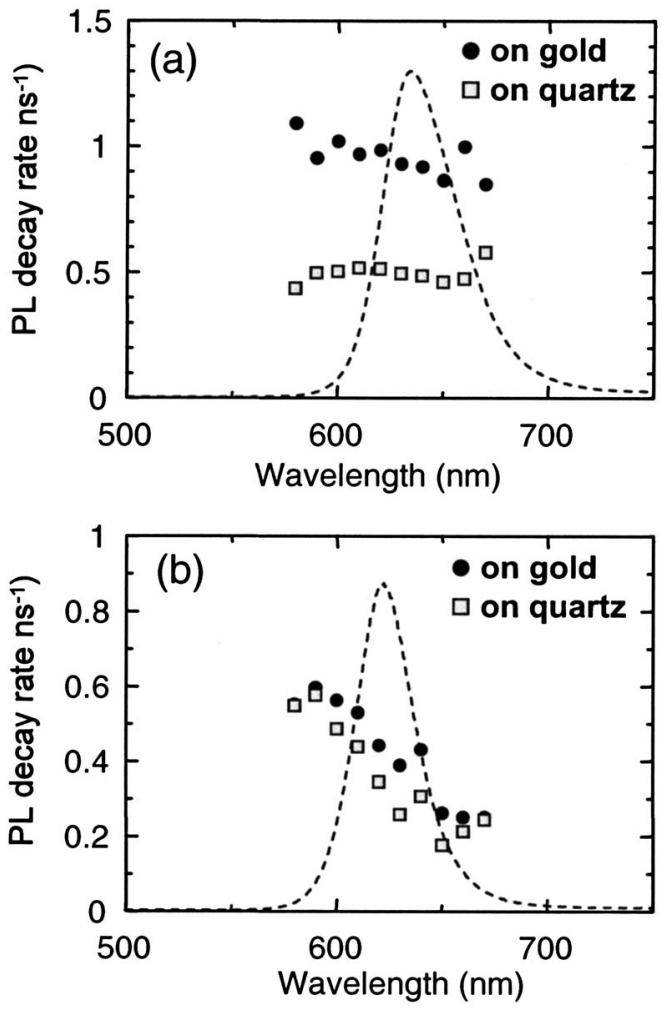

Fig. 4. Wavelength dependences of the PL decay rates for (a) CdSe and (b) CdSe/ZnS nanocrystals on gold and quartz. Dashed curves are PL spectra. 


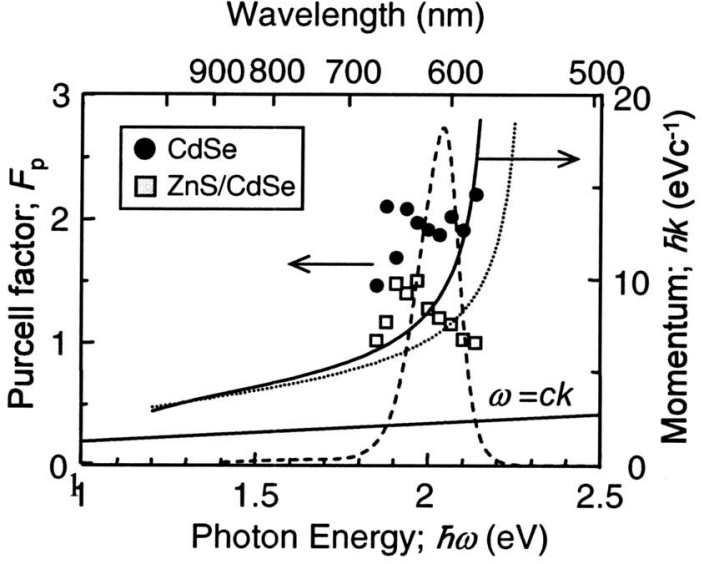

Fig. 5. Purcell enhancement factors obtained by the ratios of PL lifetimes plotted against wavelength. Solid and dashed curves are dispersion diagram of surface plasmon mode for $\mathrm{CdSe} / \mathrm{Au}$ and $\mathrm{CdSe} / \mathrm{Au}$ interface, respectively.

$$
F_{p}=\frac{k_{P L}^{*}}{k_{P L}}=\frac{k_{\mathrm{SP}}+k_{\mathrm{rad}}+k_{\mathrm{non}}}{k_{\mathrm{rad}}+k_{\mathrm{non}}} .
$$

The obtained $F_{p}$ values of CdSe and CdSe/ZnS QDs were plotted against wavelength in Fig. 5. The dispersion diagram of SP mode calculated by Eq. (1) for Au/CdSe and $\mathrm{Au} / \mathrm{ZnS}$ interfaces were also plotted. The wavelengthdependence behavior of obtained $F_{p}$ is correlated to the dispersion diagram. The SP frequency of Au/CdSe interface is well matched to the emission spectrum of QDs (dashed curve), especially at the higher energy region. The obtained $F_{p}$ values of CdSe are also increased at the higher energy region. This clear correlation supports our proposed QD-SP-coupling mechanism.

The external quantum efficiency $\left(\eta_{\text {ext }}\right)$ of emission from the light-emitting materials is given by the light extraction $C_{\text {ext }}$ and the internal quantum efficiency $\eta_{\text {int }}$, which is given by the ratio of radiative and nonradiative recombination rates as

$$
\eta_{\text {ext }}=C_{\text {ext }} \times \eta_{\text {int }}=C_{\text {ext }} \frac{k_{\text {rad }}}{k_{\text {rad }}+k_{\text {non }}} .
$$

The SP-coupling modified internal quantum efficiency $\eta_{\text {int }}{ }^{*}$ is given by

$$
\eta_{\text {int }}^{*}=\frac{k_{\mathrm{SP}}+k_{\mathrm{rad}}}{k_{\mathrm{SP}}+k_{\mathrm{rad}}+k_{\mathrm{non}}} .
$$

Therefore, if $k_{\mathrm{SP}}$ is much faster than $k_{\text {rad }}$ and $k_{\text {non }}\left(k_{\mathrm{PL}}{ }^{*}\right.$ $\left.\gg k_{\mathrm{PL}}\right), \eta_{\text {int }}{ }^{*}$ should be much larger than $\eta_{\text {int }}$. This suggests that the SP coupling can enhance the internal quantum efficiencies. ${ }^{6,13}$

There are two methods to increase $\eta_{\text {int }}$, namely, decrease $k_{\text {non }}$ or increase $k_{\text {rad. }}$. Previous work has focused on reducing $k_{\text {non }}$ by growing higher-quality crystals. Making the core/shell structure of QDs is one of these methods. The aforementioned carrier-localization effect of $\mathrm{CdSe} / \mathrm{ZnS}$ reduces $k_{\text {non }}$ and increases $\eta_{\text {int }}$. Indeed, the $\eta_{\text {int }}$ value of CdSe $(2.0 \pm 0.5 \%)$ is enhanced for a CdSe/ZnS structure $(40 \pm 10 \%)$. This 20 -fold enhancement of efficiency is based on the reducing $k_{\text {non }}$, because obtained $k_{\mathrm{PL}}$ of CdSe/ZnS are smaller than those of naked CdSe (Fig. 4). This enhancement ratio is comparable with that of the SP-coupling technique. However, the SP-coupling technique enhances not only emission intensities but also emission rates because it is based on increasing $k_{\text {rad }}$. This is an important advantage for high-speed light-emitting devices for the development of communication technology and optical computing. There have been very few studies focusing on increasing $k_{\text {rad }}$, even though that could prove to be most effective for development of high $\eta_{\text {int }}$ light emitters. The SP-coupling technique is one of the few solutions to increase $k_{\text {rad }}$.

Figure 6 shows the relationship between internal quantum efficiency $\left(\eta_{\text {int }}{ }^{*}\right)$ and $F_{p}$ with several initial values of efficiencies $\left(\eta_{\text {int }}^{0}\right)$. We calculated them by using the following relationship (6):

$$
F_{p}=\frac{1-\eta_{\text {int }}{ }^{0}}{1-\eta_{\text {int }}^{*}} .
$$

$\eta_{\text {int }}{ }^{*}$ values dramatically increase with increasing of $F_{p}$ values. The $\eta_{\text {int }}$ value of CdSe can be enhanced from $2 \%$ to $51 \%$ when $F_{p}=2$. A 26 -fold enhancement of emission is expected by this figure. This value is reasonable to the experimental value (23-fold) of the PL enhancement ratio of CdSe on gold. On the other hand, the $\eta_{\text {int }} 0$ value of $\mathrm{ZnS} / \mathrm{CdSe}$ can be enhanced from $40 \%$ to $75 \%$ when $F_{p}$ $=2$. The expected enhancement ratio is only 1.9 fold. This discrepancy should be the reason for the reduced SP enhancement of $\mathrm{CdSe} / \mathrm{ZnS}$ on gold. This suggests that the SP-coupling technique is very effective for increasing the emission efficiency of materials with low intrinsic efficiency, but not so effective for high-efficiency materials.

In Fig. $6, \eta_{\text {int }}{ }^{*}$ values are over $90 \%$ regardless of the initial values at the regions of $F_{p}>10$. This is a very useful advantage of this technique. If even high $F_{p}$ values could be achieved by well-matched SP-coupling condition, bright emission properties of materials are no longer necessary. Even when using the materials with originally very dark emissions $\left(\eta_{\text {int }}{ }^{0} \sim 0 \%\right)$, very bright emission efficiencies $\left(\eta_{\text {int }}{ }^{*} \sim 50 \%\right.$ with $F_{p}=2 ; \eta_{\text {int }}{ }^{*} \sim 90 \%$ with $F_{p}=10$ ) can be expected with this technique. However, if the $\eta_{\text {int }}{ }^{0}$ value is very high, the $\eta_{\text {int }}{ }^{*}$ value does not increase much even if $F_{p}$ value is very high. Thus this technique is not

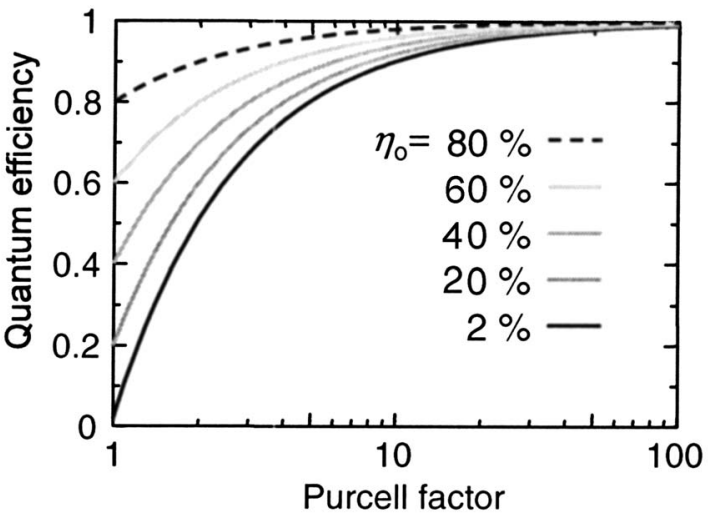

Fig. 6. Relationship between internal quantum efficiency of emission $\left(\eta_{\text {int }}{ }^{*}\right)$ and Purcell factor $\left(F_{p}\right)$ with initial quantum efficiency $\left(\eta_{\text {int }}{ }^{0}\right) 80 \%, 60 \%, 40 \%, 20 \%$, and $2 \%$. 
effective for a bright $\mathrm{ZnS} / \mathrm{CsSe}$ sample; this is a demerit of this technique. Therefore the SP-enhanced technique should be applied to various materials that suffer from low quantum efficiencies, which include the indirect semiconductors ( $\mathrm{Si}, \mathrm{Ge}, \mathrm{SiC}$, etc.), rather than bright III-V or II-IV materials. To design and fabricate even more efficient SP-enhanced optical devices for a wider spectral range, tuning of the SP mode is very important to achieve matching condition of the energy coupling. Alternatively, the SP could be geometrically tuned by fabricating nanostructures (for example, making the grating structure) or using alloys. This technique would lead the new evolution of superbright solid-state light sources, which could be very cheap to make and easy to process and would become commonly used light sources replacing traditional fluorescent tubes. The technique also suggests the use of gold as a cladding layer for colloidal quantum dots may enhance emission at certain wavelengths and could be used as an alternative to $\mathrm{ZnS}$.

\section{CONCLUSIONS}

SP coupling is one of the most interesting methods for developing efficient solid-state LEDs, as the metal can be used both as electrical contact and for exciting plasmons. We observed dramatic enhancement of both the PL intensities and decay when CdSe QDs are located on evaporated gold films. By using this technique, high-efficiency and high-speed light emission is predicted for optically as well as electrically pumped light emitters, because the SP coupling increases the internal quantum efficiency, and this mechanism is not related to the pumping method.

\section{ACKNOWLEDGMENTS}

The authors acknowledge support from the Kavli Nanoscience Institute and the AFOSR under the Plasmon MURIFA9550-04-1-0434.

\section{REFERENCES}

1. S. Nakamura and G. Fasol, The Blue Laser Diode: GaNBased Light Emitting and Lasers (Springer, 1997).

2. V. L. Colvin, M. C. Schlamp, and A. P. Ailvisatos, "Lightemitting diodes made from cadmium selenide nanocrystals and a semiconducting polymer," Nature 370, 354-357 (1994).

3. B. O. Dabbousi, M. G. Bawendi, O. Onitsuka, and M. F. Rubner, "Electroluminescence from CdSe quantum-dot/ polymer composites," Appl. Phys. Lett. 66, 3116-3118 (1995).

4. M. Bruchez Jr., M. Moronne, P. Gin, S. Weiss, and A. P. Alivisatos, "Semiconductor nanocrystals as fluorescent biological labels," Science 281, 2013-2016 (1998).

5. W. Chan and S. Nie, "Quantum dot bioconjugates for ultrasensitive nonisotopic detection," Science 281, 2016-2018 (1998).

6. K. Okamoto, I. Niki, A. Shvartser, Y. Narukawa, T. Mukai, and A. Scherer, "Surface plasmon enhanced light-emitters based on InGaN quantum wells," Nat. Mater. 3, 601-605 (2004).

7. A. Köck, E. Gornik, M. Hauser, and W. Beinstingl, "Strongly directional emission from AlGaAs/GaAs lightemitting diode," Appl. Phys. Lett. 57, 2327-2329 (1990).

8. W. L. Barnes, "Electromagnetic crystals for surface plasmon polaritons and the extraction of light from emissive devices," J. Lightwave Technol. 17, 2170-2182 (1999).

9. I. Gontijo, M. Borodisky, E. Yablonvitch, S. Keller, U. K. Mishra, and S. P. DenBaars, "Coupling of InGaN quantumwell photoluminescence to silver surface plasmons," Phys. Rev. B 60, 11564-11567 (1999).

10. J. Vuckovic, M. Loncar, and A. Scherer, "Surface plasmon enhanced light-emitting diode," IEEE J. Quantum Electron. 36, 1131-1144 (2000)

11. A. Neogi, C.-W. Lee, H. O. Everitt, T. Kuroda, A. Tackeuchi, and E. Yablonvitch, "Enhancement of spontaneous recombination rate in a quantum well by resonant surface plasmon coupling," Phys. Rev. B 66, 153305 (2002).

12. J. R. Lakowicz, "Radiative decay engineering: biophysical and biomedical applications," Anal. Biochem. 298, 1-24 (2001).

13. K. Okamoto, I. Niki, A. Scherer, Y. Narukawa, T. Mukai, and Y. Kawakami, "Surface plasmon enhanced spontaneous emission rate of InGaN/GaN quantum wells probed by time-resolved photoluminescence spectroscopy," Appl. Phys. Lett. 87, 071102 (2005).

14. T. D. Neal, K. Okamoto, and A. Scherer, "Surface plasmon enhanced emission from dye doped polymer layers," Opt. Express 13, 5522-5527 (2005).

15. B. Jensen and A. Torabit, "Refractive index of hexagonal II-VI compounds CdSe, CdS, and $\mathrm{CdSe}_{\mathrm{x}} \mathrm{S}_{1-\mathrm{x}}$," J. Opt. Soc. Am. B 3, 857-863 (1986).

16. M. Hacskaylo and C. Feldman, "Dielectric anomaly in $\mathrm{ZnS}$ films," J. Appl. Phys. 33, 3042-3045 (1962).

17. O. Kulakovich, N. Strekal, A. Yaroshevich, S. Maskevich, S. Gaponenko, I. Nabiev, U. Woggon, and M. Artemyev, "Enhanced luminescence of CdSe quantum dots on gold colloids," Nano Lett. 2, 1449-1452 (2002).

18. J.-H. Song, T. Atay, S. Shi, H. Urabe, and A. V. Nurmikko, "Large enhancement of fluorescence efficiency from CdSe/ZnS quantum dots induced by resonant coupling to spatially controlled surface plasmons," Nano Lett. 5, 1227-1561 (2005).

19. I. Gryczynski, J. Malicka, W. Jiang, H. Fischer, W. C. W. Chan, Z. Gryczynski, W. Grudzinski, and J. R. Lakowicz, "Surface-plasmon-coupled emission of quantum dots," J. Phys. Chem. B 109, 1088-1093 (2005).

20. K. Okamoto, H.-C. Ko, Y. Kawakami, and Sg. Fujita, "Timespace resolved photoluminescence from (Zn,Cd)Se-based quantum dots structures," J. Cryst. Growth 214/215, 639-645 (2000).

21. E. Purcell, "Spontaneous emission probabilities at radio frequencies," Phys. Rev. 69, 681-681 (1946). 